\title{
On the associated graded ring of a local Cohen-Macaulay ring
}

\author{
By \\ Judith D. SALLY* \\ (Communicated by Prof. Nagata Feb. 21, 1976)
}

It seldom happens that properties of a local ring $(R, \boldsymbol{m})$ are carried over to its associated graded ring $G(R)$. Here we investigate when the property of being Cohen-Macaulay is transferred from $R$ to $G(R)$.

First, we set up some notation. $(R, \boldsymbol{m})$ denotes a local Cohen-Macaulay ring of dimension $d>0$ and multiplicity $e(R) . G(R)$ denotes its associated graded ring. $G(R)=R / \boldsymbol{m} \oplus \boldsymbol{m} / \boldsymbol{m}^{2} \oplus \boldsymbol{m}^{2} / \boldsymbol{m}^{3} \oplus \cdots$. We denote the embedding dimension of $R$, that is the number of generators in a minimal basis of $\boldsymbol{m}$, by $v(\boldsymbol{m})$.

It is true that $d \leqslant v(\boldsymbol{m}) \leqslant e(R)+d-1$. The first inequality is the Krull principal ideal theorem and the second inequality is a result of Abhyankar [A]. We investigate whether $G(R)$ is Cohen-Macaulay in terms of $v(\boldsymbol{m})$. If $v(\boldsymbol{m})$ $=d, R$ is regular and $G(R)$ is also regular. The fact that $G(R)$ is Cohen-Macaulay if $v(\boldsymbol{m})=d+1$ is also well known (see, for example, [B-S]) but for the convenience of the reader we sketch a proof here. We may assume that $R$ is a complete Cohen-Macaulay local ring and thus a homomorphic image of a $d+$ 1 dimensional complete regular local ring $S$. The kernel $C$ must be a height 1 unmixed, hence principal, ideal of $S$. Say $C=f S$. Then $G(R)=G(S) /$ $\bar{f} G(S)$, where $\bar{f}$ is the initial form of $f$ in $G(S)$. Since $G(S)$ is a polynomial ring over a field, $G(R)$ is CohenMacaulay.

We will show that if $v(\boldsymbol{m})=e(R)+d-1$ then $G(R)$ is Cohen-Macaulay and that this is the only other case where $G(R)$ is Cohen-Macaulay for all such $(R, \boldsymbol{m})$.

Theorem 1. Assume that $R / m$ is an infinite field. Then there exist elements $x_{1}, x_{2}, \cdots, x_{d}$ in $\boldsymbol{m}$ such that $\boldsymbol{m}^{2}=\left(x_{1}, x_{2}, \cdots, x_{d}\right) \boldsymbol{m}$ if and only if $v(\boldsymbol{m})=e(R)+d-1$.

Proof. By the results of Northcott and Rees $[\mathrm{N}-\mathrm{R}]$ on minimal reductions of an ideal, there exist elements $x_{1}, x_{2}, \cdots, x_{d}$ in $\boldsymbol{m}$ with $\boldsymbol{m}^{n}=\boldsymbol{x}^{n+1}$ for some positive integer $n$, where $\boldsymbol{x}=\left(x_{1}, x_{2}, \cdots, x_{d}\right)$. Tensor the exact sequence

* The author was partially supported by NSF GP 29815 


$$
0 \rightarrow \boldsymbol{m} \rightarrow R \rightarrow R / \boldsymbol{m} \rightarrow 0
$$

by $R / \boldsymbol{x}$ to obtain the exact sequence:

$$
0 \rightarrow \operatorname{Tor}_{1}{ }^{R}(R / \boldsymbol{m}, R / \boldsymbol{x}) \rightarrow \boldsymbol{m} / \boldsymbol{x} \boldsymbol{m} \rightarrow R / \boldsymbol{x} \rightarrow R / \boldsymbol{m} \rightarrow 0 .
$$

Let $\lambda_{R}(A)$ denote length of the $R$-module $A$. We have that $\lambda_{R / m}\left(\operatorname{Tor}_{1}{ }^{R}(R / m\right.$, $R / \boldsymbol{x}))=d$ because $\boldsymbol{x}$ is a regular sequence. By the properties of minimal reductions, $e(R)=\lambda_{R / x}(R / x)$. Hence, $\lambda_{R / m}(\boldsymbol{m} / \boldsymbol{x} \boldsymbol{m})=e(R)+d-1$. From this it follows immediately that $v(\boldsymbol{m}) \leqslant e(R)+d-1$, and that $v(\boldsymbol{m})=e(R)+d-1$ if and only if $\boldsymbol{m}^{2}=\boldsymbol{x} \boldsymbol{m}$.

Theorem 2. If $v(\boldsymbol{m})=e(R)+d-1$, then $G(R)$ is Cohen-Macaulay.

Proof. We may assume that $R / \boldsymbol{m}$ is an infinite field. By Theorem 1 , there exist elements $x_{1}, x_{2}, \cdots, x_{d}$ in $\boldsymbol{m}$ such that $\boldsymbol{m}^{2}=\boldsymbol{x} \boldsymbol{m}$. We prove by induction on $d$, that $\bar{x}_{1}, \bar{x}_{2}, \cdots, \bar{x}_{d}$, the images of $x_{1}, x_{2}, \cdots, x_{d}$ in $G(R)$, form a regular sequence. By $[\mathrm{H}-\mathrm{R}],[\mathrm{M}-\mathrm{R}]$ this is sufficient to prove that $G(R)$ is Cohen-Macaulay. If $d=1$, we have $m^{2}=x_{1} m . \bar{x}_{1}$ is not a zero divisor for $x_{1} y \in \boldsymbol{m}^{t+1}=x_{1} \boldsymbol{m}^{t}$, for any $t>1$, implies that $y \in \boldsymbol{m}^{t}$ because $x_{1}$ is not a zero divisor in $R$. Assume that $d>1$. We first check that $\bar{x}_{1}$ is not a zero divisor in $G(R)$. If $x_{1} y \in m^{t+1}=\left(x_{1}, \cdots, x_{d}\right)^{t} \boldsymbol{m}$, where $t>1$, we must show that $y \in \boldsymbol{m}^{t}$ $=\left(x_{1}, \cdots, x_{d}\right)^{t-1} m$. $x_{1} y=g\left(x_{1}, \cdots, x_{d}\right) x_{1}+f\left(x_{2}, \cdots, x_{d}\right)$, where $g\left(x_{1}, \cdots, x_{d}\right)$ is a homogeneous polynomial of degree $t-1$ in $x_{1}, \cdots, x_{d}$ with coefficients in $\boldsymbol{m}$ and $f\left(x_{2}, \cdots, x_{d}\right)$ is a homogeneous polynomial of degree $t$ in $x_{2}, \cdots, x_{d}$ with coefficients in $\boldsymbol{m}$. Hence $h x_{1}=f\left(x_{2}, \cdots, x_{d}\right)$ with $h=y-g\left(x_{1}, \cdots, x_{d}\right)$. Since $x_{1}$, $\cdots, x_{d}$ is a regular sequence in $R$, the associated graded ring of $R$ with respect to the ideal $\boldsymbol{x}=\left(x_{1}, \cdots, x_{d}\right)$ is a polynomial ring in $d$ variables over $R / \boldsymbol{x}$. Hence $h \in\left(x_{1}, \cdots, x_{d}\right)^{t}$ and $y \in\left(x_{1}, \cdots, x_{d}\right)^{t-1} \boldsymbol{m}$. Pass to the Cohen-Macaulay ring $R /$ $x_{1} R . \quad e\left(R / x_{1} R\right)=e(R), \operatorname{dim} R / x_{1} R=d-1$ and $v\left(\boldsymbol{m} / x_{1} R\right)=v(\boldsymbol{m})-1$. The induction hypothesis applies to $R / x_{1} R$ so that $\bar{x}_{2}, \cdots, \bar{x}_{d}$ form a regular sequence in $G\left(R / x_{1} R\right)=G(R) / \bar{x}_{1} G(R)$. Hence $\bar{x}_{1}, \bar{x}_{2}, \cdots, \bar{x}_{d}$ is a regular sequence in $G(R)$.

Remark. The case $d=1$ is also proved in [D].

Corollary 3. If $e(R) \leqslant 3$, then $G(R)$ is Cohen-Macaulay.

To show that $v(\boldsymbol{m})=d, d+1$ and $e(R)+d-1$ are the only cases where $G(R)$ is Cohen-Macaulay for all local Cohen-Macaulay rings $R$, we construct a 1-dimensional local Cohen-Macaulay ring $(R, \boldsymbol{m})$ with $v(m)=d+2=e(R)+$ $d-2$ and with $G(R)$ not Cohen-Macaulay. M. Hochster showed me that if $k$ is a field, then $k\left[\left[t^{5}, t^{8}, t^{27}\right]\right]$ is an axample of a 1-dimensional complete local domain $R$ with $G(R)$ not Cohen-Macaulay. The following examples show that such a domain can be constructed for any multiplicity $e(R) \geqslant 4$. Let $k$ be a field. Let $e$ be any integer $\geqslant 4$. The conductor of the numerical semigroup generated by $e$ and $e+1$ is $(e-1) e$. Let $R=k\left[\left[t^{e}, t^{e+1}, t^{(e-1) e-1}\right]\right]$. Then $\boldsymbol{m}=$ $\left(t^{e}, t^{e+1}, t^{(e-1) e-1}\right)$ and $v(\boldsymbol{m})=3$. Now $t^{(e-1) e-1} \boldsymbol{m} \subseteq \boldsymbol{m}^{3}$; for $t^{(e-1) e-1} t^{e}=\left(t^{e+1}\right)^{e-1}$, $t^{(e-1) e-1} t^{e+1}=t^{e}$ and $\left(t^{(e-1) e-1}\right)^{2}=\left(t^{e}\right)^{e-1}\left(t^{e+1}\right)^{e-2}$. Thus the maximal homogeneous ideal of $G(R)$ belongs to 0 in $G(R)$. In particular, if $e=4$, then $R=k\left[\left[t^{4}, t^{5}\right.\right.$, 
$\left.t^{11}\right]$ has $v(\boldsymbol{m})=3=d+2=e(R)+d-2$ and $G(R)$ is not Cohen-Macaulay.

DEPARTMENT OF MATHEMATICS

NORTHWESTERN UNIVERSITY

EVANSTON, ILLINOIS 60201

U.S.A.

\section{References}

[A] S. S. Abhyankar, Local rings of high embedding dimension, Amer. J. Math. 89 (1967), 1073-1077.

[B-S] M. Boratynski and J. Swiecicka, The Hilbert-Samuel function of a Cohen-Macaulay ring, preprint.

[D] E. D. Davis, On the geometric interpretation of seminormality, preprint.

[H-R] M. Hochster and L. J. Ratliff, Jr., Five theorems on Macaulay rings, Pac. J. of Math. 44 (1973), 147-172.

[M-R] J. Matijevic and P. Roberts, A conjecture of Nagata on graded Cohen-Macaulay rings, J. Math. Kyoto Univ. 14 (1974), 125-128.

[N-R] D. G. Northcott and D. Rees, Reductions of ideals in local rings, Proc. Camb. Phil. Soc. 50 (1954), 145-158. 